\title{
Transatlantica
}

Revue d'études américaines. American Studies Journal

1 | 2017

Morphing Bodies: Strategies of Embodiment in

Contemporary US Cultural Practices

Quicksilver Brilliance: Adolf de Meyer Photographs, Metropolitan Museum of Art, The Howard Gilman Gallery, 4 décembre 2017-18 mars 2018

Commissariat : Beth Saunders, conservatrice associée au département des photographies

\section{Alice Morin}

\section{OpenEdition}

Journals

Édition électronique

URL : https://journals.openedition.org/transatlantica/9102

DOI : $10.4000 /$ transatlantica.9102

ISSN : $1765-2766$

Éditeur

Association française d'Etudes Américaines (AFEA)

Référence électronique

Alice Morin, «Quicksilver Brilliance: Adolf de Meyer Photographs, Metropolitan Museum of Art, The

Howard Gilman Gallery, 4 décembre 2017-18 mars 2018 », Transatlantica [En ligne], 1 | 2017, mis en

ligne le 13 octobre 2018, consulté le 20 mai 2021. URL : http://journals.openedition.org/transatlantica/ 9102 ; DOI : https://doi.org/10.4000/transatlantica.9102

Ce document a été généré automatiquement le 20 mai 2021.

\section{(c) $(1)$}

Transatlantica - Revue d'études américaines est mise à disposition selon les termes de la licence Creative Commons Attribution - Pas d'Utilisation Commerciale - Pas de Modification 4.0 International. 


\section{Quicksilver Brilliance: Adolf de Meyer Photographs, Metropolitan Museum of Art, The Howard Gilman Gallery, 4 décembre 2017-18 mars 2018}

Commissariat : Beth Saunders, conservatrice associée au département des photographies

\section{Alice Morin}

1 Loin des grandes expositions rétrospectives pour lesquelles le Met est connu, les deux salles de Quicksilver Brilliance: Adolf de Meyer Photographs offrent au visiteur une sélection de moments choisis de l'œuvre du photographe. Organisée par le département de photographie du Met, cette exposition prend d'emblée le parti d'un propos sélectif, en présentant quelques quarante travaux dans une muséographie sobre et classique.

Le texte introductif rappelle que le baron Adolf de Meyer (1868-1946) est né à Paris et a reçu une éducation européenne, avant d'intégrer le "monde » londonien par son mariage en 1899 avec Olga Caracciolo (réputée fille naturelle du roi Edward VII). Le couple, cosmopolite, s'installe définitivement à New York lors de la Première guerre mondiale. Meyer (anobli baron peu après son mariage) est donc membre d'un cercle international aristocratique du tournant $\mathrm{du} \mathrm{xx}^{\mathrm{e}}$ siècle, au sein duquel musiciens, écrivains et personnalités mondaines se fréquentent et où leurs pratiques se répondent. C'est sa vie sociale brillante qu'il fixe sur pellicule, mais sa production est loin cependant d'être documentaire. Ses mises en scènes sont savamment composées, souvent théâtrales.

De fait, les tirages, ouvrages et extraits de publications présentés attestent d'une production variée mais d'une grande cohérence esthétique. Une partie provient de la donation Alfred Stieglitz - ami et premier collectionneur de Meyer. Cette relation marque leur adhésion commune aux groupes d'avant-garde de la photographie américaine. Meyer, qui expose dans les galeries Photo-Secession entre 1900 et le début des années 1910, entretient aussi avec Stieglitz une correspondance nourrie sur sa 
pratique. Dans son introduction au catalogue de l'exposition sur de Meyer conçue par Anne Ehrenkranz en 1994 à l'International Center of Photography, Willis Hartshorn affirme : "In London around the turn of the century, de Meyer's social position and apparent financial independance may have worked to his advantage, but it was the sophisticated beauty of his still lifes and portraits that gave him access to the most advanced photographic circles»(Ehrenkranz, 1994, 3). La lecture de son œuvre proposée par cette exposition s'effectue au prisme de sa double appartenance à ces cercles qui se croisent.

\section{Meyer, entre pictorialisme et cosmopolitisme mondain}

4 L'exposition s'ouvre sur le smoking de gala du photographe ${ }^{1}$ qui fait allusion à son cosmopolitisme mondain, attesté plus loin par les portraits de ses mécènes américains comme la socialite Rita de Acosta Lydig, français comme le comte Etienne de Beaumont ou britanniques comme l'égérie littéraire Lady Otteline Morrell.

5 Le vêtement est encadré par deux portraits emblématiques : d'une part un close-up de 1903 aux intonations intimes par Gertrude Käsebier, portraitiste de studio établie à Newport, Rhode Island et d'autre part une mise en scène costumée "à la mexicaine " par Sarah Choates Sears (1905). Ces deux images permettent de souligner les liens que toutes deux, comme Meyer, entretiennent avec les groupes «Photo-Secession» et "The Linked Ring », qui souhaitaient promouvoir la photographie artistique, des ÉtatsUnis à l'Europe, au tout début du Xx $x^{e}$ siècle.

Des études pictorialistes affichent aussi l'intérêt de Meyer pour des objets de son quotidien luxueux, sublimés par ses agencements, notamment The Silver Cap et Water Lillies présentée sous forme d'une épreuve au platine mais aussi dans un exemplaire relié de Camera Works de 1912. Dans ces images la texture prime, sensible dans les jeux d'une lumière tamisée dirigée sur un bonnet de soie argentée ou sur les reflets de l'eau d'un vase posé sur son support, jouant ainsi sur la transparence, élément-clé de ses compositions.

7 Également réparties entre portraits et natures mortes, les œuvres exposées dans cette section sont riches d'influences visuelles érudites. L'esthétique raffinée met l'accent sur la beauté des tissus et leurs ornementations, renvoyant à la peinture flamande du XVII ${ }^{\mathrm{e}}$ siècle. Le sujet des photographies présentées (souvent des femmes somptueusement vêtues) préfigure aussi l'incursion de l'auteur dans la photographie de mode, que développe la suite de l'exposition.

\section{Une pratique photographique «brillante » aux influences multiples}

8 La deuxième salle propose une sélection parmi les nombreux portraits de sa muse, sa femme Olga, qui fut sa partenaire artistique plus qu'amoureuse (Meyer étant homosexuel, comme l'était peut-être aussi Olga), ainsi que des natures mortes prises lors de leurs voyages en Europe et en Asie autour en 1900. Ces tirages, tantôt enveloppés d'une brume créant un effet de flou autour de mises en scènes aux riches costumes (Olga de Meyer, 1912), tantôt étonnamment sobres dans leurs lignes pour certains paysages (View through a Garden Window, 1900), témoignent d'un orientalisme 
commun à une avant-garde inspirée de Whistler et à une élite internationale fascinée par l'exotisme. Un travail permanent, dans la droite ligne pictorialiste, sur la lumière souvent indirecte et le cadrage sensible (souvent horizontal), met une nouvelle fois la texture à l'honneur. Riches étoffes et larges surfaces mates dialoguent ainsi dans des compositions soignées, comme par exemple dans le portrait d'olga de Meyer, Japan de 1900.

9 Un curieux autoportrait complète l'aperçu de cette période : Self Portrait in India (1900) présente l'artiste à l'arrière-plan de la composition. Il est encadré par les montants d'une large baie vitrée, laissant apparaitre une végétation luxuriante à laquelle répond une décoration intérieure typiquement coloniale. L'horizontalité de la photographie, son manque de perspective (car le fond en est flou) et la nonchalance de la pose de Meyer semblent attester d'une volonté d'effet total, qui fait primer la représentation d'une scène sur celle d'un individu.

10 Cette quête esthétique se double d'un vif intérêt pour les techniques permettant d'obtenir ce résultat, comme en atteste l'accrochage du fac simile d'un autochrome (Tamara Karsavina, ca. 1908), dont le cartel rappelle que Meyer cherchait à introduire la couleur dans son travail. Plusieurs des lettres de l'artiste à Stieglitz témoignent ainsi de sa frustration à devoir circonscrire son travail au noir et blanc, ce qui explique qu'il embrasse avec enthousiasme cette technologie innovante qui permet d'illuminer par derrière une épreuve sur plaque de verre colorée. En outre, cette représentation de la danseuse russe Tamara Karsavina au repos dans un jardin anglais amorce une série de portraits d'artistes et de mondains, membres de son cercle familier.

11 Dans la continuité esthétique de ses œuvres précédentes, ces tirages marquent cependant une évolution dans la pratique de Meyer qui s'essaie au travail de commande. Toujours référentielles ${ }^{2}$, ces photographies, qui incluent également un portrait de Josephine Baker (1925-26) font le lien avec son travail pour la presse de mode $^{3}$, puisque de nombreuses épreuves présentées ici sont tirées des pages de magazines - le portrait de Rita de Acosta Lydig est d'abord paru dans Vogue, tandis qu'un article signé Meyer sur les fastueuses fêtes du comte de Beaumont figurera dans Harper's Bazaar en 1923.

12 Meyer est toujours préoccupé par la reconnaissance de la photographie comme une forme d'art à part entière, et c'est sous son impulsion que les traditionnelles illustrations de ces publications sont progressivement remplacées par des photographies (Seebohm, 1982, 164-180). Réciproquement, on peut observer que l'œuvre photographique du baron s'enrichit au contact de ses expérimentations graphiques. L'exemple le plus frappant en la matière est The New Hat Called Violette Worn by The Honorable Mrs Reginald Fellowes (1928), qui inaugure une série de photographies de mode dans lesquelles des modèles, aux visages de plus en plus lisses, se détachent nettement de fonds travaillés (abstraits ou au contraire surchargés), sous l'effet d'une lumière crue. La dernière photographie présentée est d'ailleurs celle d'un mannequin de cire (Mannequin in Suzanne Talbot Hat, 1929, en couleur) souligne le penchant " surréaliste » du photographe pour les masques et les poupées (Brown, 2009).

13 Complétant cet accrochage classique, une vitrine présente des exemplaires reliés de magazines, mettant en évidence l'influence de son travail sur la photographie de mode. Meyer y mène des expérimentations techniques sur les jeux de lumière (plongeante ou plus diffuse), de pose et de cadrage. Son style onirique et précieux s'adapte au luxe de cet univers. 


\section{Texture et mouvement : photographier la danse}

d'envergure : Prélude à l'Après-midi d'un faune, édité en mille exemplaires à partir d'une étude réalisée sur le célèbre ballet éponyme de Nijinski à Paris en 1912. Un exemplaire relié de l'ouvrage est exposé, accompagné de six phototypes.

Dans la vitrine, le livre fait la part belle aux mises en page audacieuses, s'appuyant une fois encore sur de nouvelles techniques. La torsion du corps du danseur costumé, que fixe la photographie, manifeste la fascination esthétique qu'il exerce sur Meyer. L'artiste parvient également à rendre, voire à intensifier l'univers immersif que créent la mise en scène élaborée de Nijinski et les costumes de Léon Bakst. La transparence de ceux-ci est traduite sur la page, faisant allusion à la sensualité de l'argument (alors scandaleuse). Cette sensualité est palpable, et encore augmentée par les plis de l'écharpe tenue par le faune. L'ensemble donne à cette illustration du moment culminant de la chorégraphie une saisissante texture.

Les six phototypes au mur, qui sont également des pages tirées de l'ouvrage, manifestent aussi cette texture donnée au mouvement, aussi bien dans les portraits de groupe qu'individuels. Les corps et les étoffes se découpent nettement sur fond champêtre - une netteté accentuée par le procédé d'impression photomécanique obtenu à partir de plaques. Le rendu, proche de la gravure et duplicable en grandes quantités, est d'une grande finesse dans les détails, soulignant la gracilité de poses inspirées des motifs de vases grecs antiques. Meyer capture ainsi un moment de la vie artistique du tout-Paris de l'avant-guerre et retranscrit les expérimentations de son temps entre danse, musique et décors qui ont nourri son œuvre.

Ce dernier volet, consacré à la danse, semble résumer sa philosophie esthétique. L'ensemble de l'exposition propose ainsi quelques pistes, explications biographiques ou filiations stylistiques, pour appréhender une œuvre rarement montrée aux États-Unis ou à l'étranger (Meyer meurt ruiné et oublié en 1946, et son style est vite remplacé par une sobriété inspirée de la Straight Photography dans les pages des magazines, où Steichen lui succède). Les choix muséaux, qui invitent à une contemplation sensorielle de sa production, mettent néanmoins en lumière l'importance historique de ce corpus éminemment représentatif d'une époque opulente et extravagante, révolue dès le début des années 1930. 
Gertrude Käsebier, Baron Adolf de Meyer, 1903

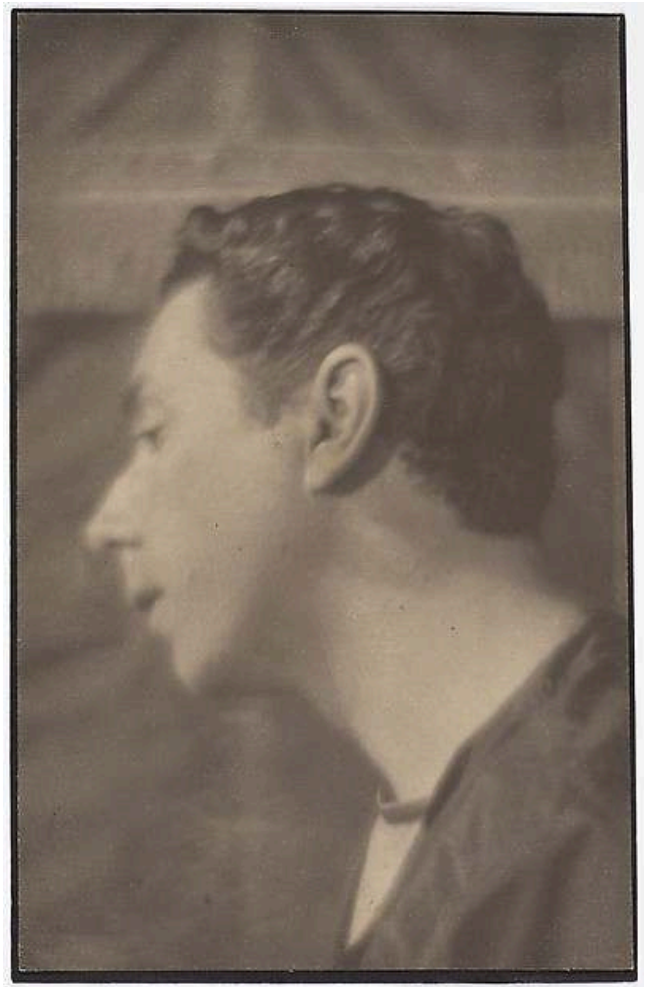

Épreuve au platine, 22,1 x 14,2 cm, The Metropolitan Museum of Art (c) Gilman Collection, Museum Purchase, 2005, URL 
Sarah Choate Sears, A Mexican, 1905

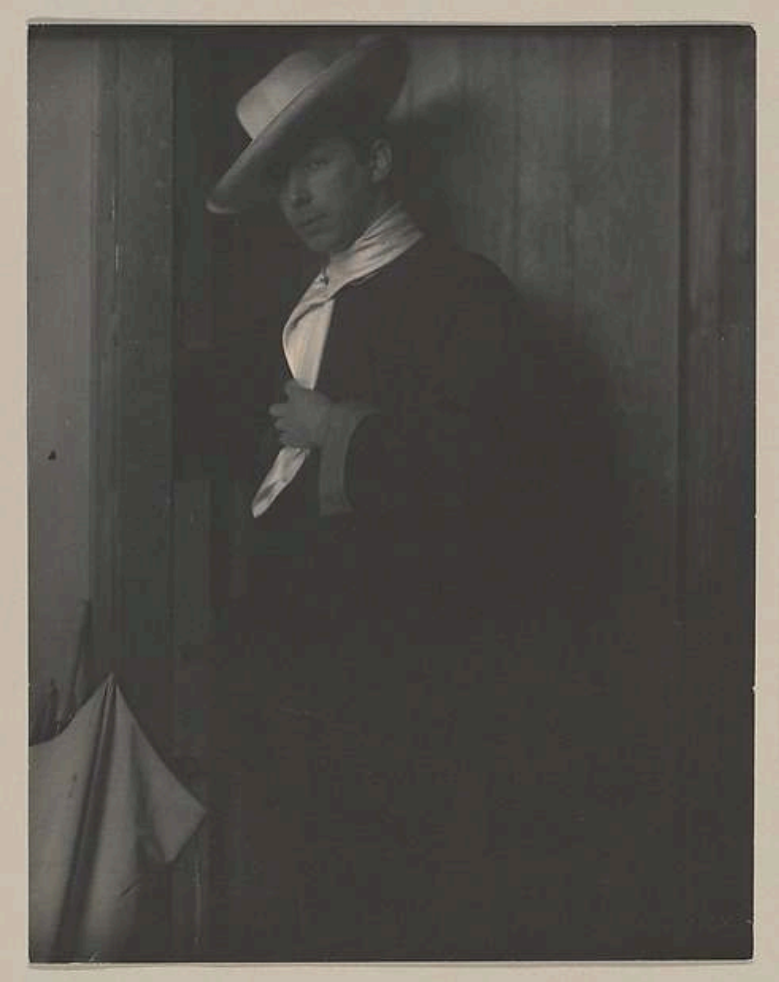

Épreuve au platine, 24,2 x 18,7 cm, The Metropolitan Museum of Art (c) Alfred Stieglitz Collection, 1933, URL 
Adolf de Meyer, The Silver Cap, ca. 1909

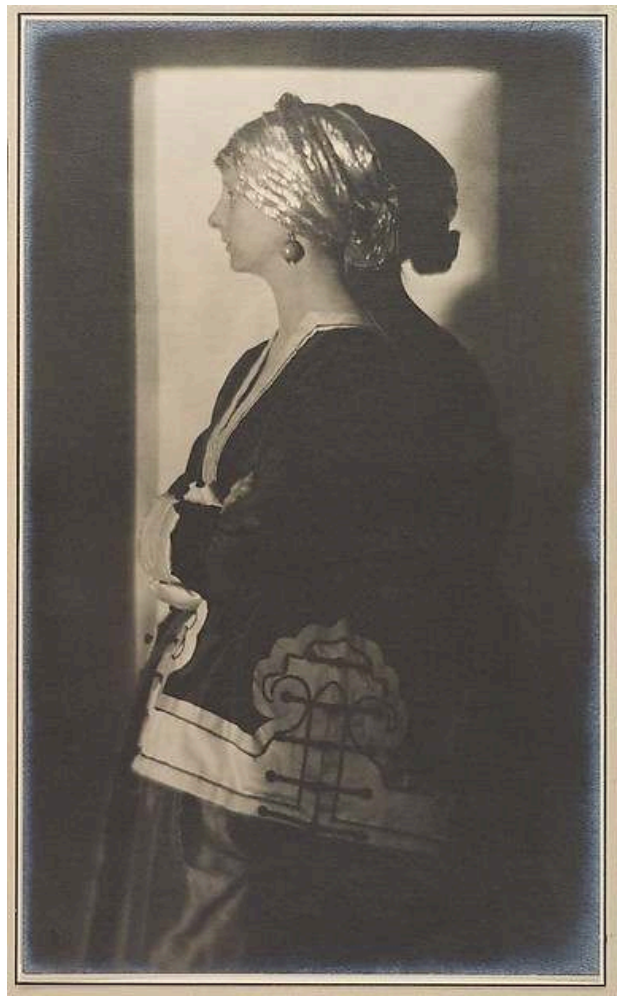

Épreuve argentique de 1912, 45,7 × 27,6 cm, The Metropolitan Museum of Art (c) Alfred Stieglitz Collection, 1933, URL

Adolf de Meyer, Water Lilies, ca. 1906

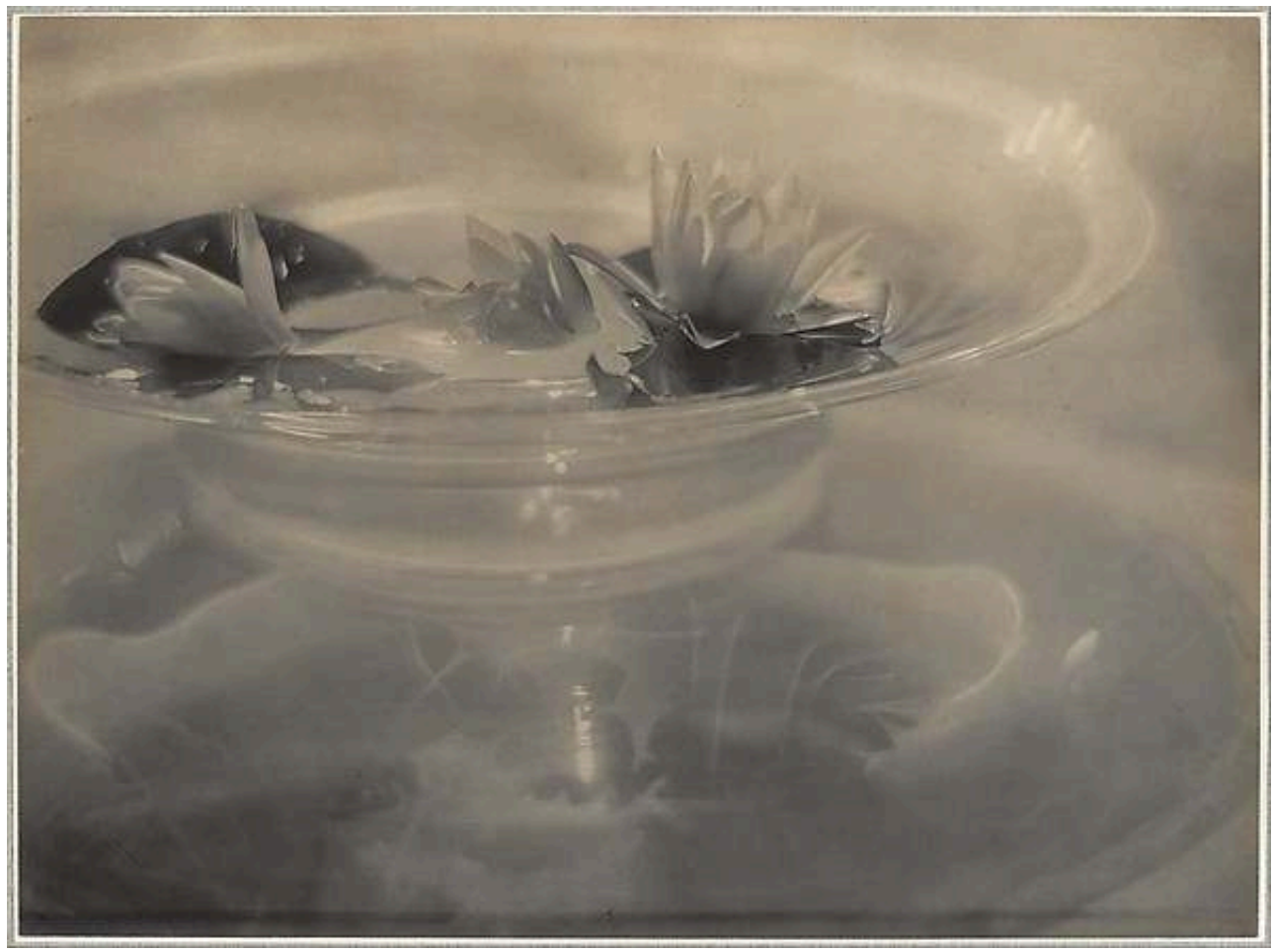

Épreuve au platine de 1912, 45,7 × 27,6 cm, The Metropolitan Museum of Art (c) Alfred Stieglitz Collection, 1933, URL 
Adolf de Meyer, Olga de Meyer, ca. 1912

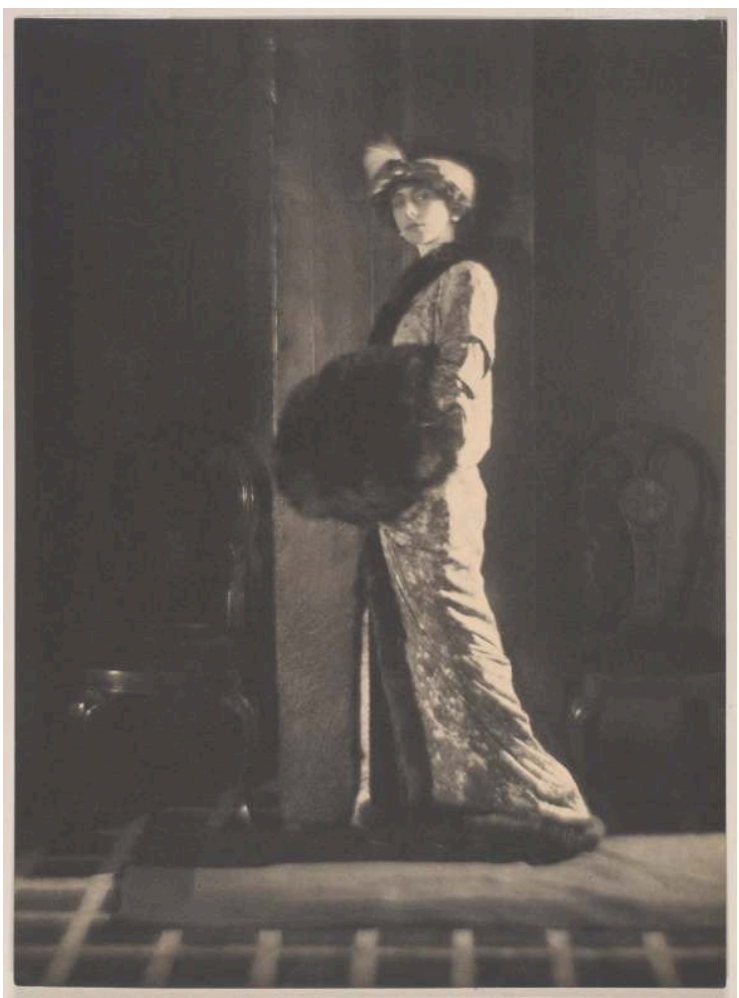

ÉPReUVe AU PLATINE, 22,4 x 16,4 cM, THE METROPOLITAN MUSEUM OF ART

(C) Gift of Paul F. Walter, 2009, URL

Adolf de Meyer, Untitled [View Through the Window of a Garden, Japan], 1900

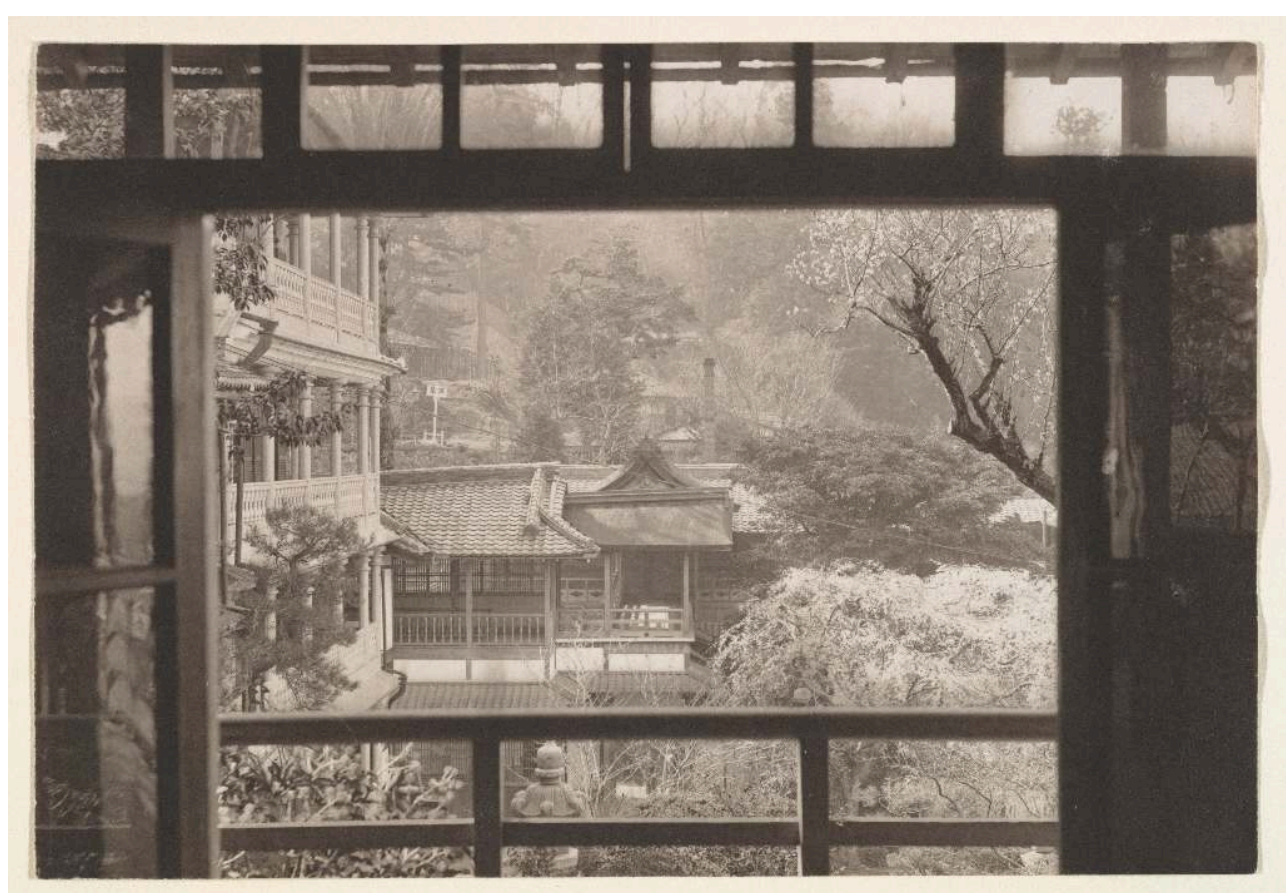

ÉPREUVe AU PLATINe, $\mathbf{1 3 , 8} \times \mathbf{2 0} \mathbf{2 0}$ cM, THE METROPOLITAN MUSEUM OF ART

(C) Purchase, Mrs. Jackson Burke Gift, 1981, URL 
Adolf de Meyer, Olga de Meyer, Japan, 1900

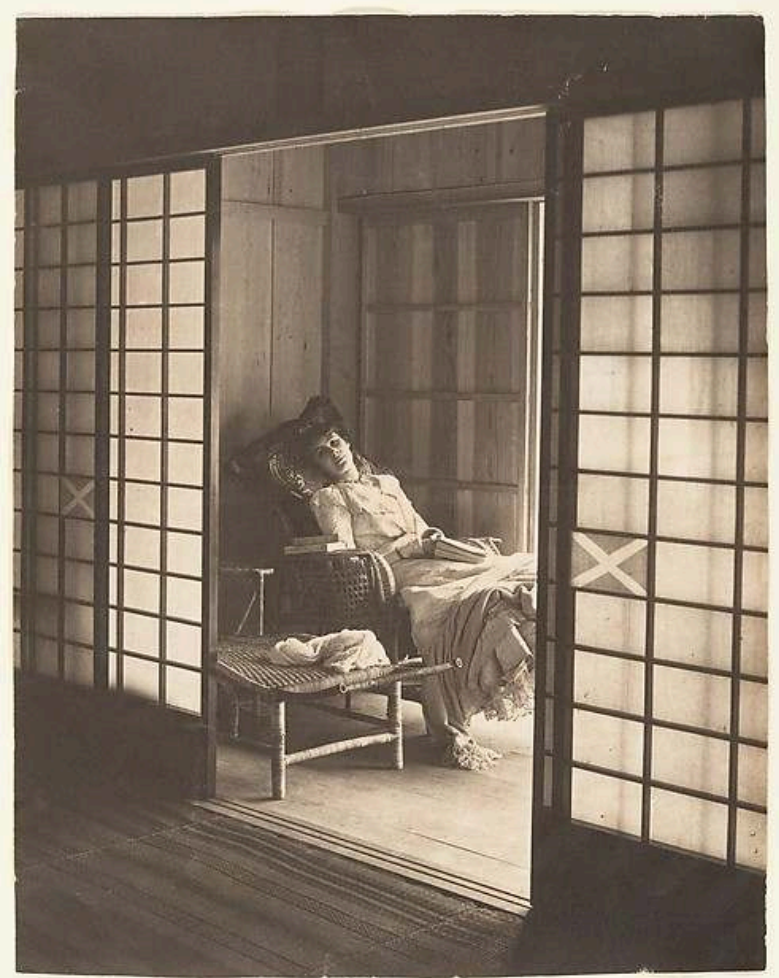

ÉPREUVE AU PLATINE, 19,3 x 15,1 CM, THE METROPOLITAN MUSEUM OF ART

(c) Purchase, Mrs. Jackson Burke Gift, 1981, URL 
Adolf de Meyer, Untitled [Self-Portrait in India], 1900

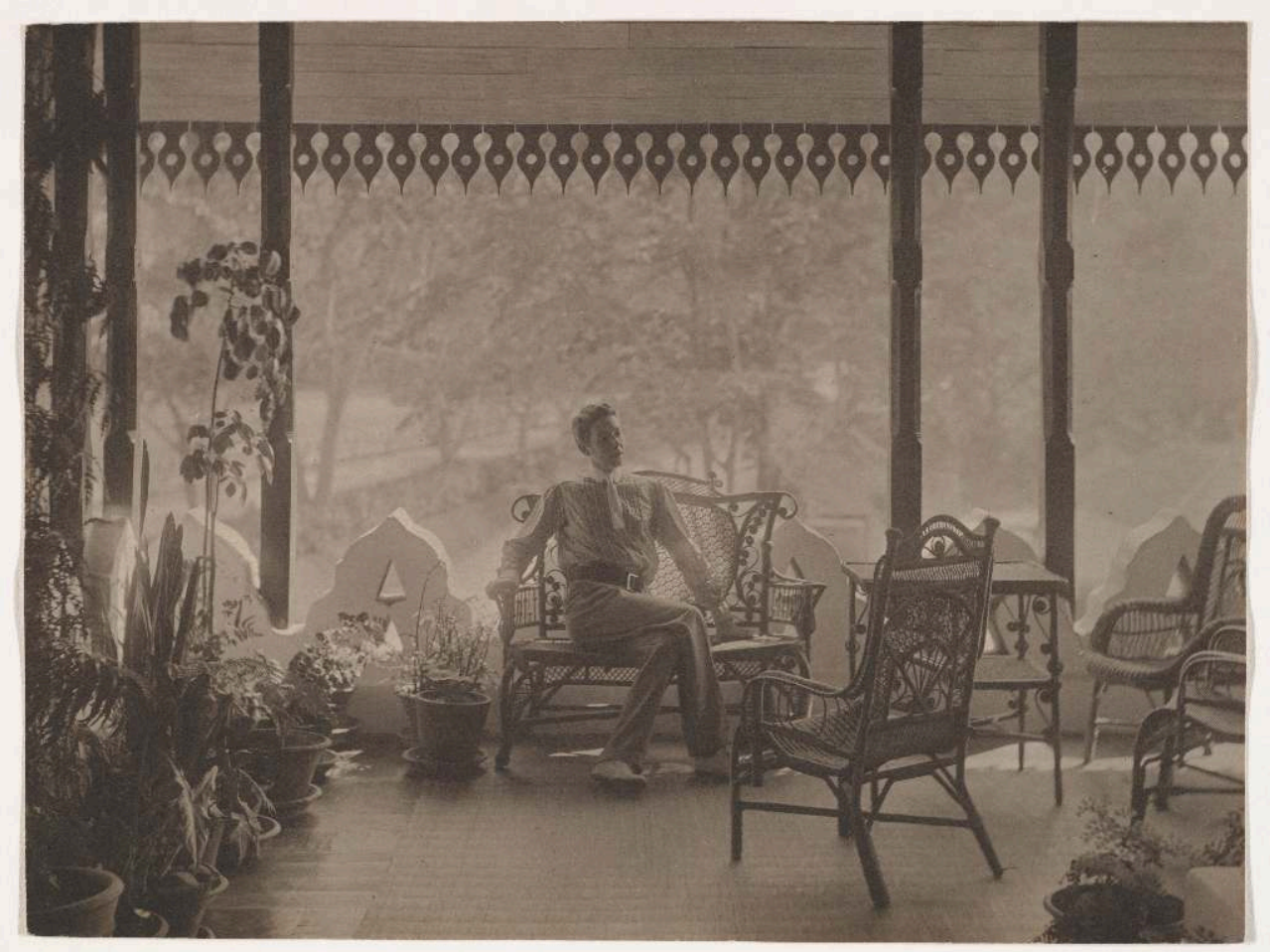

ÉPREUVE AU PLATINE, 14,8 x 19,8 cM, THE METROPOLITAN MUSEUM OF ART

(C) Gift of Isaac Lagnado, 1995, URL

Adolf de Meyer, Tamara Karsavina, ca. 1908.

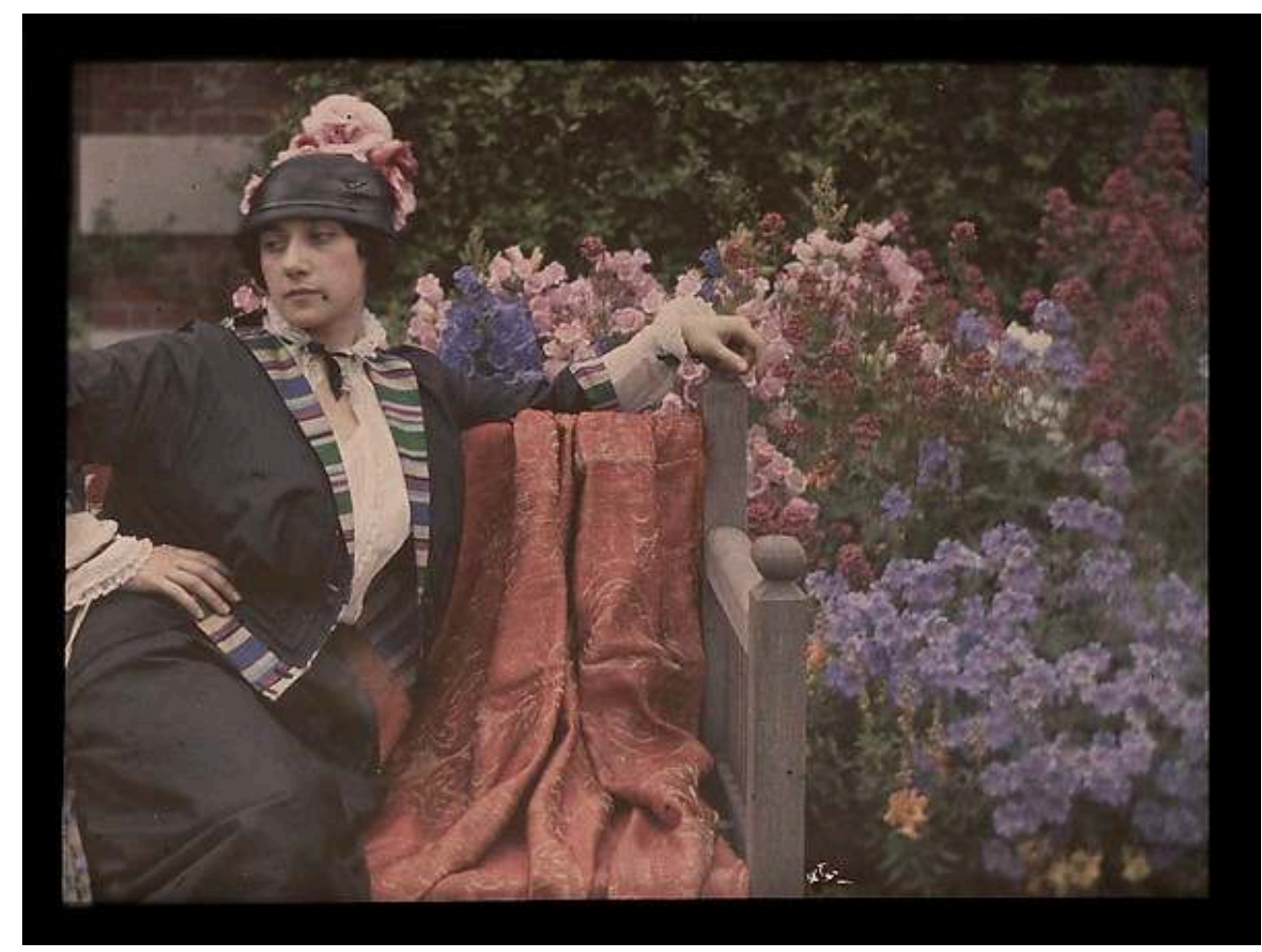

Autochrome, $9 \times 11,9 \mathrm{~cm}$, The Metropolitan Museum of Art

(c) Gilman Collection, Purchase, Harriette and Noel Levine Gift, 2005, URL 
Adolf de Meyer, Josephine Baker, 1925-26

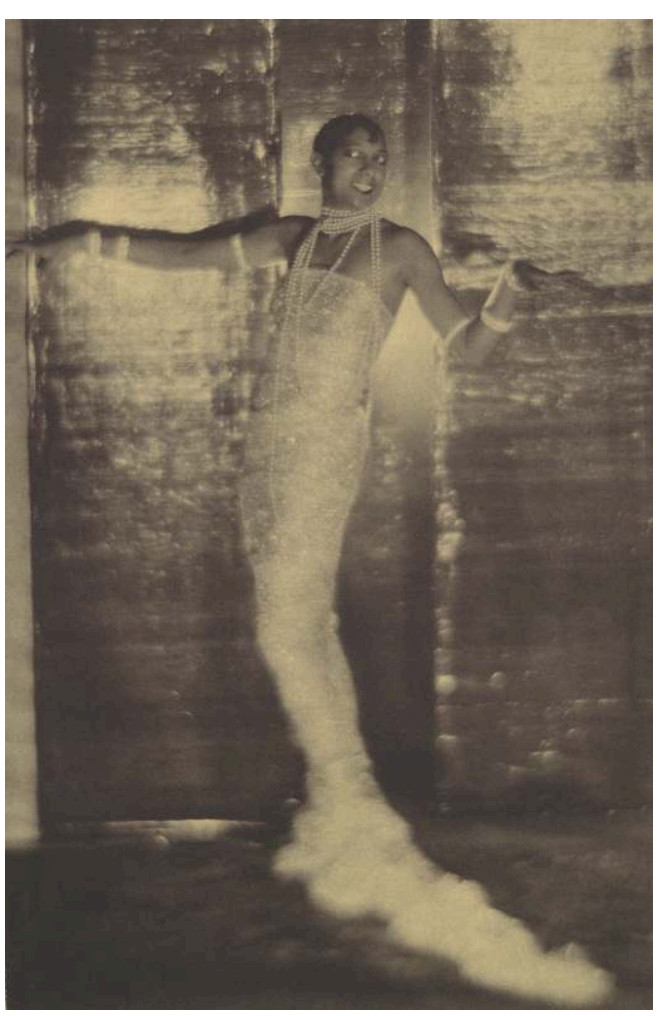

ÉPREUVE AU CARBONe, 45,2 X 29,5 CM, THE METROPOLITAN MUSEUM OF ART

(C) Ford Motor Company Collection, Gift of Ford Motor Company and John C. Waddell, 1987, URL 
Adolf de Meyer, Rita de Acosta Lydig, 1917

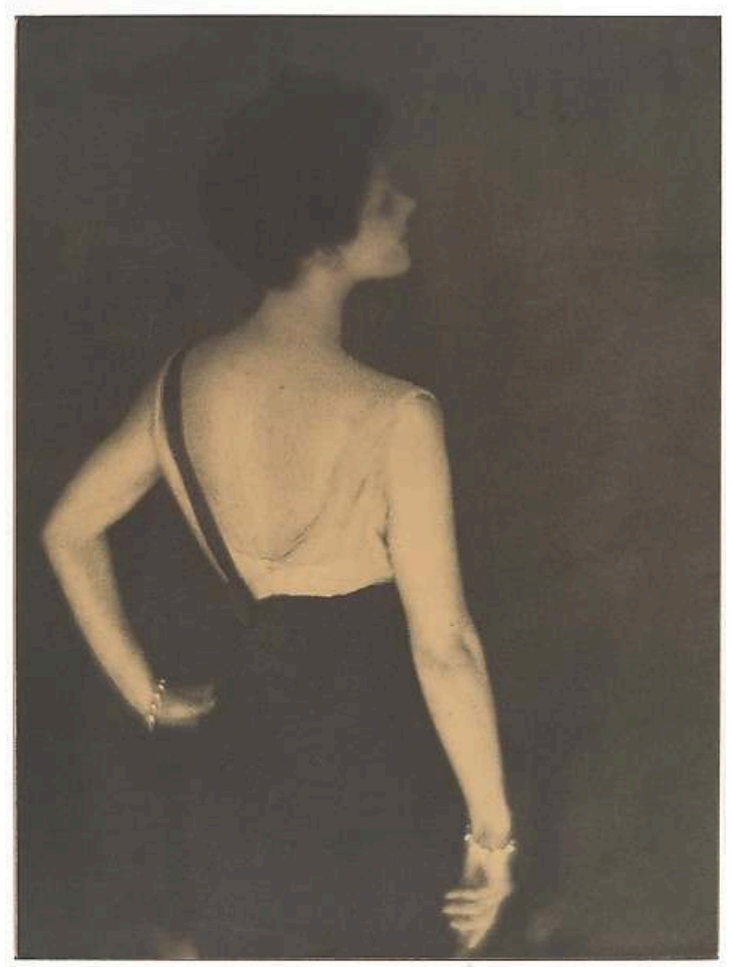

ÉPREUVe AU PLATINE, 41,5 X 30,9 cM, THE METROPOLITAN MUSEUM OF ART

(C) Gift of Mercedes de Acosta, 1952, URL 
Adolf de Meyer, Etienne de Beaumont, ca. 1923

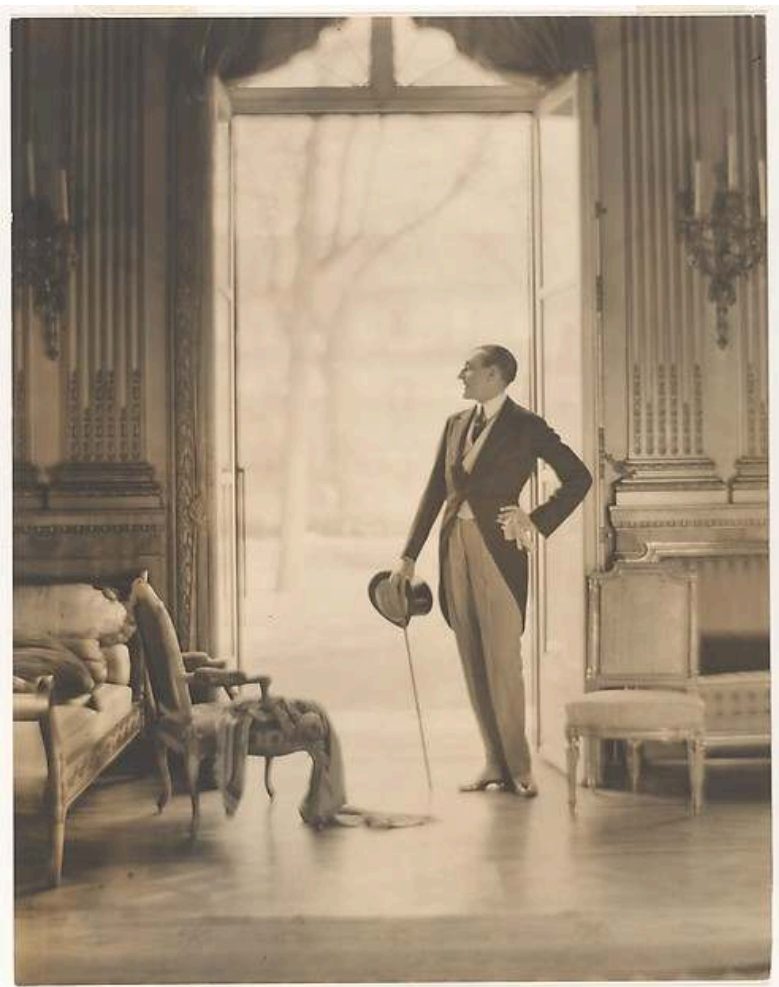

ÉPREUVE ARgENTIQUE, 23,7 X 18,6 CM, THE METROPOLITAN MUSEUM OF ART

(C) Gift of Paul F. Walter, 2009, URL 
Adolf de Meyer, The New Hat Called Violette Worn by The Honorable Mrs. Reginald Fellowes, 1924

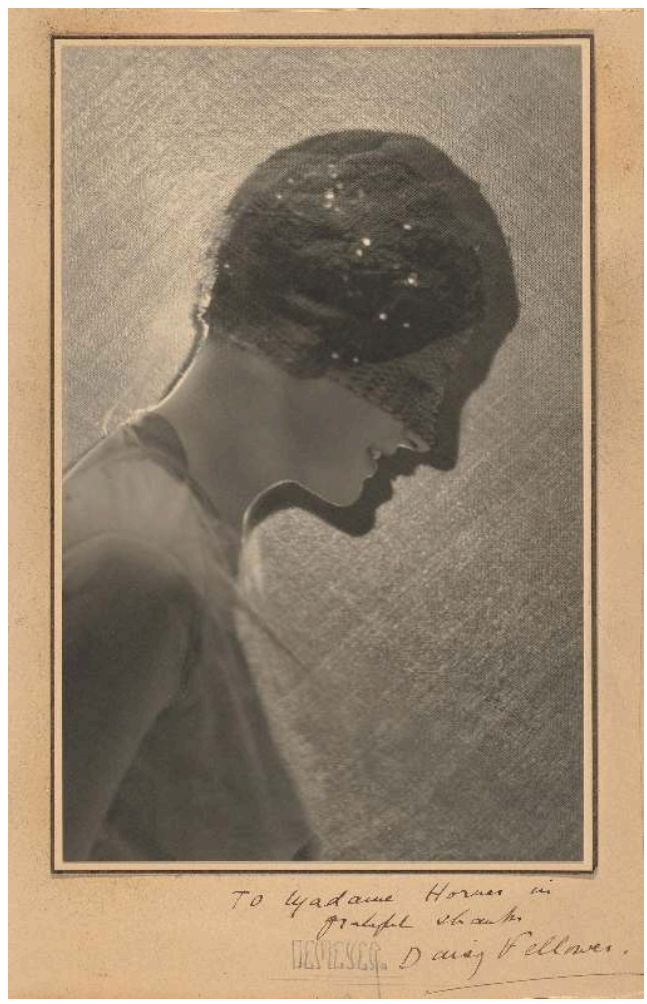

ÉPReUVe ARgentique, 22,8 x 14,6 cM, THE MEtropolitAN MUSEUM OF ART

(c) Gift of Paul F. Walter, in memory of Christopher Hemphill, 1987, URL 
Adolf de Meyer, Untitled [Mannequin in Suzanne Talbot hat], 1929

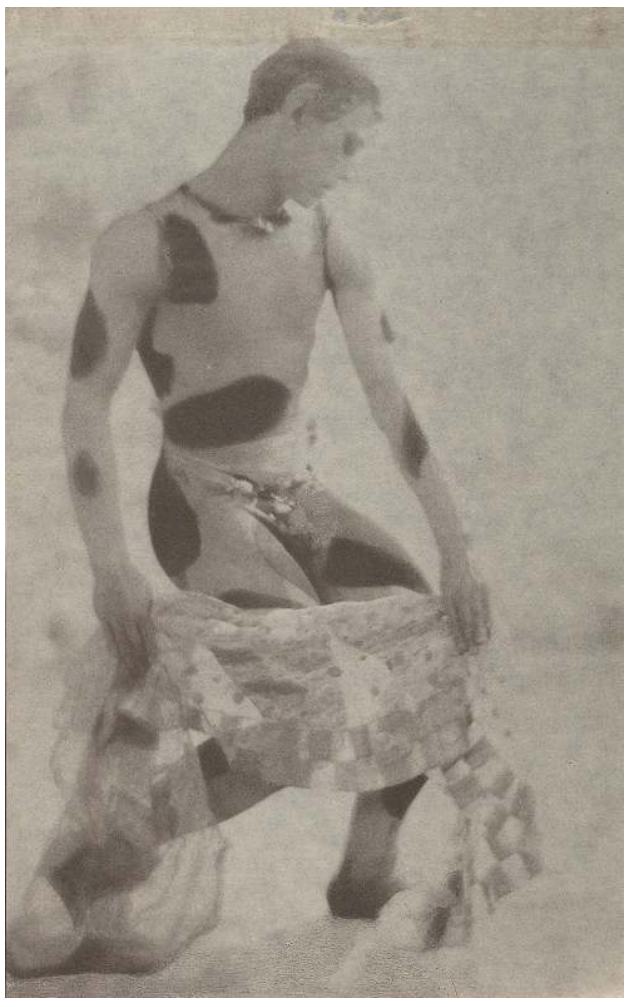

ÉPREUVE CARBRO-TRICHROME, 20,9 X 16,8 CM, THE METROPOLITAN MUSEUM OF ART

(C) Gilman Collection, Museum Purchase, 2005, URL 
Adolf de Meyer, Le Prélude à l'Après-Midi d'un Faune, 1914

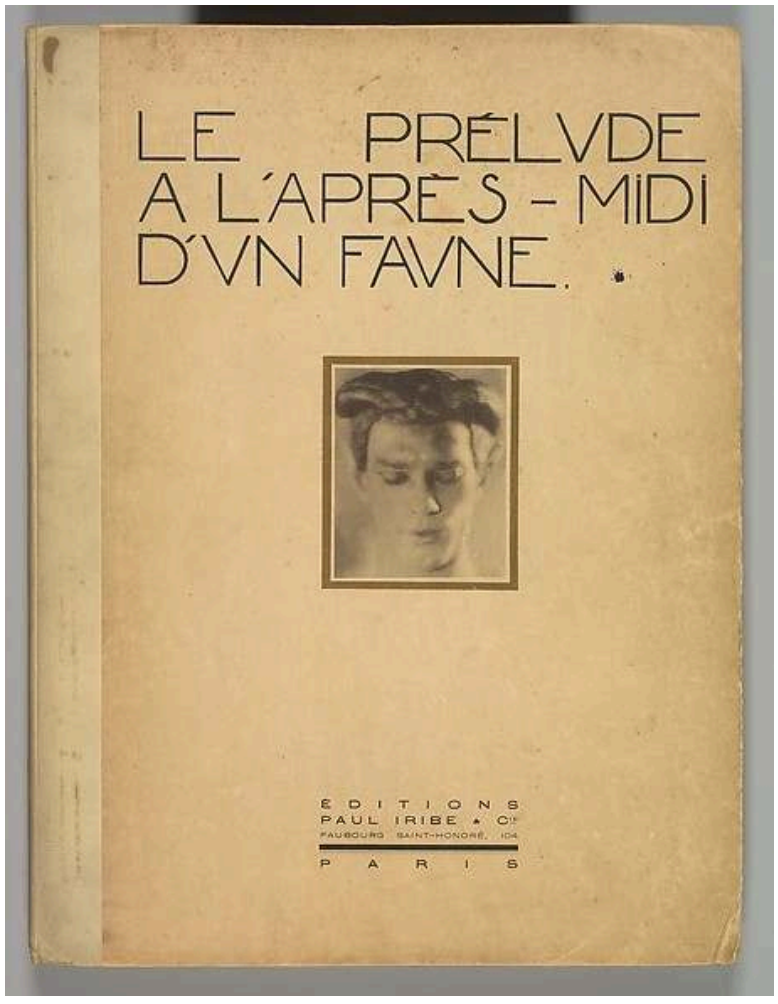

PHOTOTYPES, ALBUM : $\mathbf{3 8 , 7}$ × 29,5 CM, THE METROPOLITAN MUSEUM OF ART

(C) Gilman Collection, Museum Purchase, 2005, URL

Adolf de Meyer, Untitled [Image from "Prélude à l'Après-Midi d'un faune"], 1912

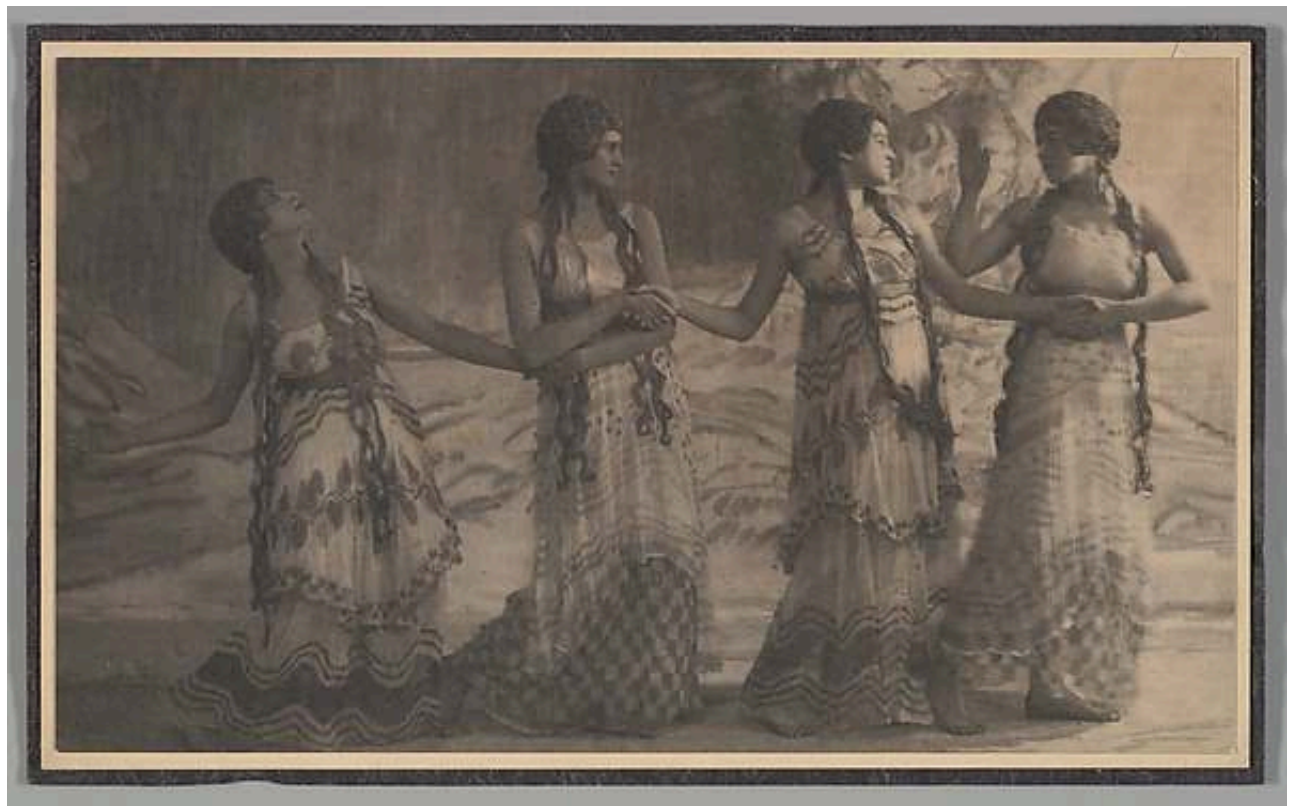

\section{ÉPREUVE AU PLATINE, $11,5 \times 18,8$ cM, THE METROPOLITAN MUSEUM OF ART}

(C) Gilman Collection, Purchase, Mrs. Walter Annenberg and The Annenberg Foundation Gift, 2005, URL 


\section{BIBLIOGRAPHIE}

EHRENKRANZ, Anne, Willis HARSHORN et John SZARKOWSKI, A Singular Elegance: The Photographs of Baron Adolph de Meyer, San Francisco, Chronicle Books avec l'International Center of Photography, 1994.

BROWN, Elspeth H., « De Meyer at Vogue: Commercializing Queer Affect in First World War-era Fashion Photography ", Photography and Culture 2/3, 2009, 253-273.

SEEBOHM, Caroline, The Man Who was Vogue: The Life and Times of Condé Nast, Londres, Weidenfeld \& Nicolson, 1982.

\section{NOTES}

1. Meyer est qualifié "d'arbitre du style" par un cartel qui rappelle qu'il écrivit aussi des chroniques vestimentaires pour Vogue et Harper's Bazaar, avec qui il collabore plus tard dans sa carrière.

2. Le portrait de Rita de Acosta Lydig, ca. 1917, par exemple, rappelle irrésistiblement la Madame X de John Singer Sargent (1884). Le tableau est accroché tout près, dans l'Aile américaine, et Lydig fut aussi l'amie du peintre.

3. De 1913 à 1921, de Meyer devient photographe attaché à Vogue et Vanity Fair, puis, de retour à Paris, directeur artistique de Harper's Bazaar dans les années 1920.

\section{INDEX}

Thèmes : Trans'Arts

\section{AUTEUR}

\section{ALICE MORIN}

Université Sorbonne Nouvelle-Paris 3 